\title{
Gaussian diffusion sinogram inpainting for X-ray CT metal artifact reduction
}

\author{
Chengtao Peng ${ }^{1}$, Bensheng Qiu' ${ }^{1}$ Ming Li ${ }^{2}$, Yihui Guan ${ }^{3}$, Cheng Zhang ${ }^{2}$, Zhongyi Wu ${ }^{2}$ and Jian Zheng ${ }^{2 *}$
}

\author{
*Correspondence: \\ zhengj@sibet.ac.cn \\ ${ }^{2}$ Medical Imaging \\ Department, Suzhou Institute \\ of Biomedical Engineering \\ and Technology, Chinese \\ Academy of Science, \\ Suzhou, China \\ Full list of author information \\ is available at the end of the \\ article
}

\begin{abstract}
Background: Metal objects implanted in the bodies of patients usually generate severe streaking artifacts in reconstructed images of X-ray computed tomography, which degrade the image quality and affect the diagnosis of disease. Therefore, it is essential to reduce these artifacts to meet the clinical demands.

Methods: In this work, we propose a Gaussian diffusion sinogram inpainting metal artifact reduction algorithm based on prior images to reduce these artifacts for fanbeam computed tomography reconstruction. In this algorithm, prior information that originated from a tissue-classified prior image is used for the inpainting of metalcorrupted projections, and it is incorporated into a Gaussian diffusion function. The prior knowledge is particularly designed to locate the diffusion position and improve the sparsity of the subtraction sinogram, which is obtained by subtracting the prior sinogram of the metal regions from the original sinogram. The sinogram inpainting algorithm is implemented through an approach of diffusing prior energy and is then solved by gradient descent. The performance of the proposed metal artifact reduction algorithm is compared with two conventional metal artifact reduction algorithms, namely the interpolation metal artifact reduction algorithm and normalized metal artifact reduction algorithm. The experimental datasets used included both simulated and clinical datasets.
\end{abstract}

Results: By evaluating the results subjectively, the proposed metal artifact reduction algorithm causes fewer secondary artifacts than the two conventional metal artifact reduction algorithms, which lead to severe secondary artifacts resulting from impertinent interpolation and normalization. Additionally, the objective evaluation shows the proposed approach has the smallest normalized mean absolute deviation and the highest signal-to-noise ratio, indicating that the proposed method has produced the image with the best quality.

Conclusions: No matter for the simulated datasets or the clinical datasets, the proposed algorithm has reduced the metal artifacts apparently.

Keywords: Metal artifact reduction, Sinogram inpainting, Gaussian diffusion, Prior image, $X$-ray $C T$

\section{Background}

The use of X-ray computed tomography (CT) in the clinical setting has lasted for several decades and has considerably helped disease diagnosis and therapy. However, metallic implants in the bodies of patients usually produce severe streaking artifacts, 
which will obscure crucial information and reduce image quality. The academic community believes the explanation of this phenomenon is beam hardening $[1,2]$. Due to the pleochroism of the X-ray energy spectrum, the attenuation coefficient of the high energy X-ray is smaller than the low energy X-ray. That is to say, when an X-ray passes through body of a patient containing metal, the low energy photons are absorbed and output mostly contains high-energy photons, thus the high-energy photons detected by detector become richer. Obviously, the conventional algorithm that is widely used in CT image reconstruction, namely the filtered back-projection (FBP) reconstruction algorithm [3], fails to reconstruct the image clearly in this situation due to inaccurate projection data. To solve the problem of severe streaking artifacts, many metal artifact reduction (MAR) algorithms have been proposed.

Presently, the most commonly used MAR algorithm is the interpolated sinogram inpainting MAR algorithm, aimed to interpolate missing projection data using neighboring data by replacing the corrupted data from the information of nearby slices and then using FBP to reconstruct the image. This type of algorithm has an appealing merit in its computation speed. Kalender et al. [4] have proposed to interpolate corrupted projection data via linear interpolation (LI) from their neighbors. The problem is that the transition between the original and interpolated projections is not sufficiently smooth, causing severe secondary artifacts. In addition to LI, cubic spline [5, 6], wavelet interpolation [7], and total variation [8-10] sinogram inpainting techniques are also used in MAR. Meanwhile, other methods try to replace the corrupted projections by relying on nearby regions or corresponding prior projections. For example, Chen et al. [11] proposed to restore corrupted projections using nearby regions via the similarity criterion. First, they segmented implanted metals and metal artifacts from the original image. Second, they restored the corrupted projections relying on nearby projections via the similarity criterion. The results of this method are largely dependent on the quality of the segmentation. However, when the metal artifacts contain complicated bone structures, this approach is likely to perceive the bone as bright artifacts, therefore causing a blurring of the bone structures in the corrected image. Tang et al. [12] restored the corrupted projections by replacing the metal slices with corresponding prior projections. In this method, the prior image is reconstructed with a down-sampled sinogram. This method sacrifices high-frequency details so that the final corrected image is relatively vague, and the details of the tissue cannot be assessed. Like Tang et al., Bal and Spies [13] have proposed to replace the metal slices in the projection domain by prior images in the same region. The problem with this method is that the prior projections over the metal trace are not well-fitted with their neighbors in the originals, thus producing new artifacts. To solve the problem of fitness in the interpolated sinogram inpainting technique, Meyer et al. [14] have proposed the normalized metal artifact reduction (NMAR) algorithm. They first normalized the original sinogram using a prior sinogram, the corrupted data were linearly interpolated and the final completed sinogram was obtained by denormalizing the interpolated sinogram. Because the NMAR algorithm still relies on LI, when used in an image with complicated structures, such as a tooth image, secondary artifacts would increase. Additionally, in the NMAR algorithm it is hard to avoid severe streaking secondary artifacts when the target contains more than one metal because it cannot interpolate the metal trace accurately. 
Based on the forward sinogram inpainting MAR framework, in this paper we propose a Gaussian diffusion sinogram inpainting technique based on a prior image to reduce metal artifacts in a Bayesian framework. In this work, the optimization target is to minimize prior energy. To recover an uncorrupted image from an incomplete dataset, the sparsity of the target image is improved by subtracting the prior image. Additionally, the Gaussian diffusion function is applied to diffuse energy to recover missing projections. The Gaussian diffusion process is realized via a gradient descent algorithm. The final target image is reconstructed using the FBP algorithm. Compared with the algorithms listed in the last paragraph, this proposed method does not show the smoothness problem and can be effective for CT images that contain several metal objects. In this work, the performance of the proposed algorithm is compared with the LI and NMAR algorithms using both simulation and clinical studies. The evaluation criteria include image quality and quantitative analysis within the region of interest, all of which demonstrate that our method performs better than the other two MAR algorithms.

\section{Methods}

\section{Problem formulation and optimization approach}

Let $x$ denote the uncorrupted projection dataset, let $y$ represent the dataset with the metal projection data removed, let $\Omega$ represent the metal trace set (metal-corrupted projection region), and let $n$ represent an unknown noise. We can then formulate the following model:

$$
y=H x+n
$$

where $H$ is an operator that removes the metal-corrupted projections from $x$.

In the classical least squares (LS) approach $[15,16]$, the estimator is chosen to minimize noise:

$$
\text { (LS) : } \hat{x}_{L S}=\underset{x}{\arg \min }\|y-H x\|^{2}
$$

However, in this work the matrix $H$ is ill-conditioned, thus the LS solution usually has a huge norm and is meaningless. We then follow the Bayesian approach [17] whose optimization target in this work is to maximize the posterior probability of $x$ given $y$, which is defined as:

$$
P(x / y) \propto P(y / x) P(x)
$$

$P(y / x)$ is defined as:

$$
P(y / x) \sim e^{-\|y-H x\|^{2}}
$$

In this framework, the target $x$ is regarded as a stochastic quantity with a prior density, and:

$$
P(x) \sim e^{-U(x)}
$$

where $U(x)$ is the prior energy. Actually, this imposes the prior information onto the estimation. Now, Eq. (3) can be reformulated as follows: 


$$
P(x / y) \sim e^{-\left(\|y-H x\|^{2}+U(x)\right)}
$$

Next, the optimization target is to minimize the posterior energy, which is formulated as:

$$
\hat{x}=\underset{x}{\arg \min }\left(\|y-H x\|^{2}+U(x)\right)
$$

In this formulation (7), the first part measures the difference between $x$ and $y$, and the second part restricts $x$ to the prior information. In this work, with the decrease of noise, $y$ is approximately equal to $H x$, so the first term in (7) is close to zero. Next, the problem defined in (7) is redefined as a constrained optimization problem:

$$
\hat{x}=\underset{x}{\arg \min } U(x), \quad \text { subject to } y=H x
$$

To solve the problem of (8), we bring in the diffusion equation: [16]

$$
I_{s}=f(s) \cdot \nabla I
$$

where the symbol $\nabla$ is a derivative matrix, formed by the first-order forward difference in this work. $I$ represents the diffusion target, and $I_{s}$ is the result after diffusion. $f(s)$ is a Gaussian diffusion function: [19, 20]

$$
f(s)=e^{-\frac{s^{2}}{2 \delta^{2}}}
$$

where $s=\left\|\nabla x_{p}\right\|, x_{p}$ is the sinogram of the prior image, called a prior sinogram. The parameter $\delta$ determines the level of diffusion.

According to [18], the diffusion process can be observed as a gradient descent of the prior energy, and the gradient descent algorithm is:

$$
-\lambda U^{\prime}(x)=-\lambda \nabla^{T}\left(f\left(\left\|\nabla x_{p}\right\|\right)\right) \nabla\left(x-\mu x_{p}\right)
$$

where the parameter $\mu$ weighs the effect of the prior sinogram, and $\lambda$ is the descent speed factor.

In Eq. (11), we introduce $x_{p}$ for two reasons: first, to improve the sparsity of the target sinogram; second, to obtain the diffusion objective.

After the step of gradient descent, the algebraic reconstruction technique (ART) is applied to reduce the projection onto the convex set (POCS): [21]

$$
\operatorname{proj}(\tilde{x})=\tilde{x}+H\left(x_{o r i}-\bar{x}\right)
$$

where $x_{\text {ori }}$ is the original sinogram, and $\bar{x}$ is the sinogram after the step of gradient descent.

In Table $1, x^{-1}$ and $x^{0}$ are set equal to $x_{\text {ori }}$, the step size $\lambda$ is set to 0.03 and $\delta=4$, according to our experience and numerous experiments. The matrix $H$ is exploited to remove the metal trace, and it is constructed according to the position of the missing projections: the value in the position of the metal in the projection domain is set to zero, and the value in other areas is set to one. What calls for special attention is that the 
Table 1 The Gaussian diffusion sinogram inpainting MAR algorithm

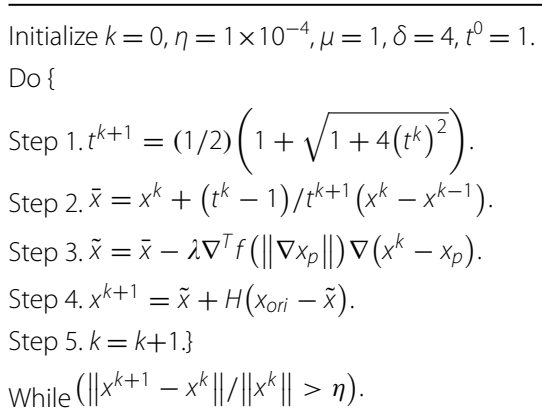

operator $H$ multiplies others is multiplying the corresponding elements between two matrices. Step 1 and step 2 are quoted from the literature [16]. The stopping criterion of the iteration is defined when the relative difference between $x^{k+1}$ and $x^{k}$ blows the tolerance $\left(\left\|x^{k+1}-x^{k}|| /|| x^{k}\right\|<\eta, \eta=1 \times 10^{-4}\right)[16,17]$.

Figure 1 provides the diagram of the proposed algorithm. In this diagram, the corresponding sinograms are produced by forward projection. Using a simple thresholding technique, the metal-only image is obtained, and the CT value for the metal is set to $3000 \mathrm{HU}$.

Concerning the prior image, $\mathrm{Li}$ et al. proposed an image post-processing strategy to create the prior image. First, they segmented the metal, and then a prior image was

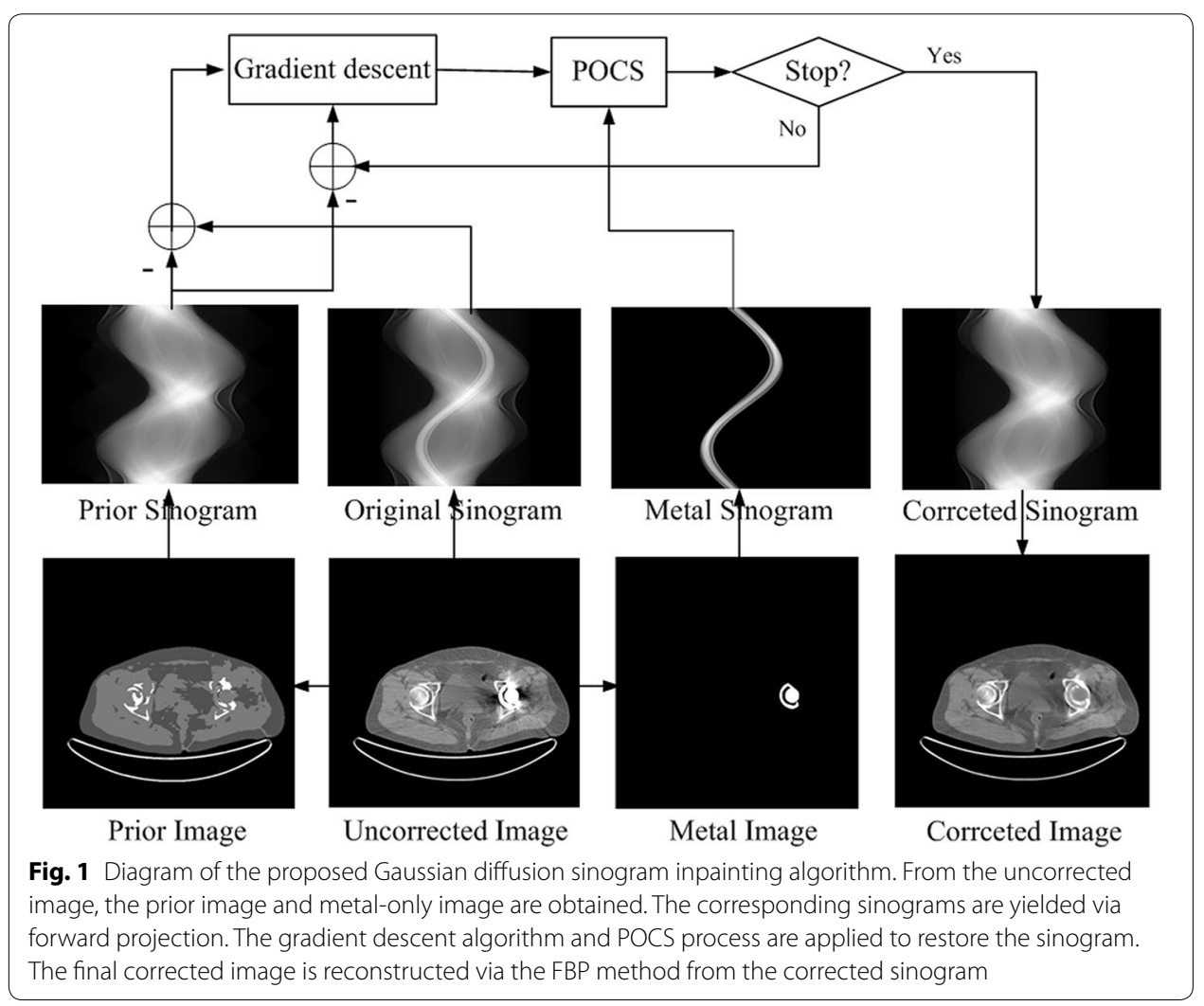


produced via an edge-preserving filter and a recovery procedure of the adjacent anatomical structures [22]. Meyer et al. [14] applied a simple thresholding method to segment air, soft tissue, and bone after the image was filtered by Gaussian. Next, the CT values for a portion of air and soft tissue were set to -1000 and $0 \mathrm{HU}$, respectively. Bone pixels kept their values because of the inherent variability of the bone densities. Different from Bal and Spies [13] who set the values of air and soft tissue with their own average CT numbers, in this work we obtained the prior image by the method of [14].

\section{Experiment materials}

The performance of the proposed algorithm is tested on both the simulated and clinical datasets, and the results are compared with two conventional algorithms (LI and NMAR). In the simulated studies, the jaw and hip phantom are applied, and the phantom size is $200 \mathrm{~mm} \times 200 \mathrm{~mm}$. In the simulation, we suppose that the process of the detector receiving photons can be modeled by the Poisson distribution [23]:

$$
I_{i}=\text { Poisson }\left\{I_{0} \cdot \exp \left(-\int_{L_{i}} u\left(l, E_{k}\right) d l\right)+S_{k}^{i}\right\}+\operatorname{Normal}\left(0, \delta_{e}^{2}\right)
$$

where $I_{i}$ is the number of photons received by the detector, $I_{0}$ is the number of photons emitted, $L_{i}$ is the X-ray path, $u$ is the attenuation coefficient of tissue, $S_{k}^{i}$ is the statistical scattering photon number, and $\delta_{e}^{2}$ is the noise variance. After logarithmic processing for the result of (13), the projection that contains noise and beam hardening is obtained. In this work, we set $E_{k}=60 \mathrm{keV}, I_{0}=5.0 \times 10^{5}$ for the hip phantom and $I_{0}=5.0 \times 10^{6}$ for jaw phantom, $S_{k}^{i}=150, \delta_{e}^{2}=10$.

The clinical dataset includes four patients: a patient with a single metal tooth, a patient implanted with two metal teeth, a patient with a single hip prosthesis and a patient with multiple metals implanted in the vertebrae.

In this work, to acquire artificial projection data under conditions close to the actual acquisition, we consider the fan-beam geometry of the simulated single-slice CT scanner with 1024 detector channels, 720 angular samples over an $360^{\circ}$ orbit, and the CT image resolution is $512 \times 512$ pixels. All of the $\mathrm{CT}$ images are processed on a PC workstation (Intel Core 3 CPU $3.60 \mathrm{GHz}$ processor and 4096 Mb RAM) by MATLAB 2010.

\section{Evaluation criteria}

The performance of the Gaussian diffusion sinogram inpainting algorithm was compared with the LI and NMAR algorithms subjectively and objectively. We subjectively evaluated the image quality for the proposed algorithm and the two other compared algorithms. We objectively evaluated the performance using the signal-to-noise ratio (SNR) and the normalized mean absolute deviation (NMAD) for the corrected images:

$$
\begin{aligned}
& S N R=10 \lg \left(\frac{\sum_{i, j}\left(u_{i, j}^{\text {truth }}\right)^{2}}{\sum_{i, j}\left(u_{i, j}-u_{i, j}^{\text {truth }}\right)^{2}}\right) \\
& N M A D(\%)=100 \times \frac{\sum_{i, j}\left|u_{i, j}-u_{i, j}^{\text {truth }}\right|}{\sum_{i, j}\left|u_{i, j}^{\text {truth }}\right|}
\end{aligned}
$$


where $u_{i, j}$ represents the corrected image, and $u_{i, j}^{\text {truth }}$ is the ideal image. Generally, SNR measures the anti-noise performance of the algorithm, and its value is greater, the algorithm produces a better quality image; NMAD measures the difference between result image and ideal image, and its value is smaller, the algorithm produces a result that is much closer to the ideal image. Besides, the mean pixel value and the standard deviation of different region of interests (ROIs) in each resulted image are analyzed.

For the clinic datasets, the ROI is defined on uncorrected images containing the metallic implants, and it is magnified for observing clearly. Besides, the reference images of the clinic datasets is hard to define, thus, we evaluated the performance only by the image quality.

\section{Results}

In this section, the results corrected by the LI, NMAR and proposed algorithms for the simulation and clinical datasets are presented. We first analyzed the simulated results, and the clinical part was analyzed subsequently.

Figure 2 shows the results for the jaw phantom with two metal dental fillings. Figure $2 \mathrm{a}$ is the true image, which contains no artifacts. Figure $2 \mathrm{~b}$ is the uncorrected image with severe streaking metal artifacts. However, Fig. 2c-e are the corrected images produced by LI, NMAR and the proposed algorithm. Clearly, the original metal artifacts are successfully reduced by each method. Unfortunately, the LI MAR algorithm causes severe secondary artifacts, which blur the tissue around the metal. NMAR and the proposed algorithms reduce the artifacts with fewer secondary artifacts than LI, and with regard to the entire picture, the proposed algorithm has a better result than NMAR, especially

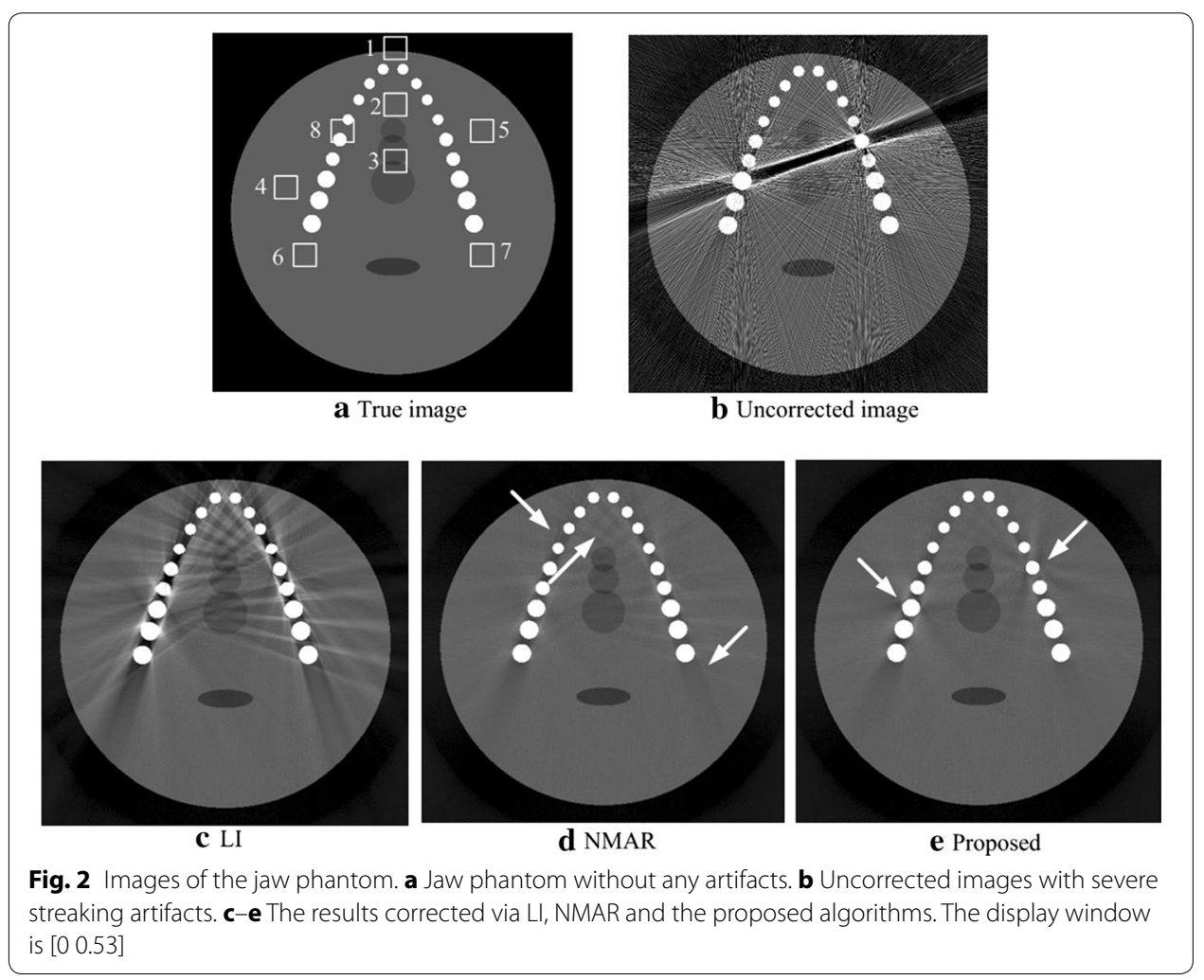


in the area between the teeth (as indicated by the arrows in Fig. 2d). However, in the proposed algorithm result, some slight artifacts are introduced around the metals, indicated as arrows in Fig. 2e primarily because the prior image was not adequate.

Table 2 shows the quantitative evaluation of experimental results. From the table, the NMAD of the proposed algorithm is smaller than those of the LI and NMAR algorithms, indicating that the image corrected by the proposed algorithm is closer to that of the true image. While the largest SNR indicates that the proposed algorithm has a better effect on suppressing the metal artifact and noise, thus producing a higher quality image.

Figure 3 compares the results for the hip phantom with a double-metal prosthesis. Figure $3 \mathrm{a}$ is the reference image with no artifacts and noise. Figure $3 \mathrm{~b}$ is the uncorrected image, as observed the severe beam harden artifacts between the metals. Figure $3 \mathrm{c}-\mathrm{e}$ are the images corrected by the LI, NMAR and proposed algorithms. All of the MAR algorithms have a good effect on reducing the metal artifacts, while the LI method introduces many residual artifacts and blurs the bone structures. Compared with LI, NMAR has a better quality image but some residual artifacts persist. Regarding the proposed algorithm, the image only introduces slight secondary artifacts, which are indicated by

Table 2 Quantitative evaluation of the experimental results for the jaw phantom

\begin{tabular}{|c|c|c|c|c|c|c|c|}
\hline \multicolumn{2}{|c|}{ Uncorrected } & \multicolumn{2}{|l|}{ LI } & \multicolumn{2}{|c|}{ NMAR } & \multicolumn{2}{|c|}{ Proposed } \\
\hline $\begin{array}{l}\text { SNR } \\
\text { (dB) }\end{array}$ & $\begin{array}{l}\text { NMAD } \\
\text { (\%) }\end{array}$ & $\begin{array}{l}\text { SNR } \\
\text { (dB) }\end{array}$ & $\begin{array}{l}\text { NMAD } \\
\text { (\%) }\end{array}$ & $\begin{array}{l}\text { SNR } \\
\text { (dB) }\end{array}$ & $\begin{array}{l}\text { NMAD } \\
(\%)\end{array}$ & $\begin{array}{l}\text { SNR } \\
\text { (dB) }\end{array}$ & $\begin{array}{l}\text { NMAD } \\
(\%)\end{array}$ \\
\hline 12.14 & 57.27 & 22.97 & 14.37 & 27.23 & 9.49 & 27.54 & 9.34 \\
\hline
\end{tabular}

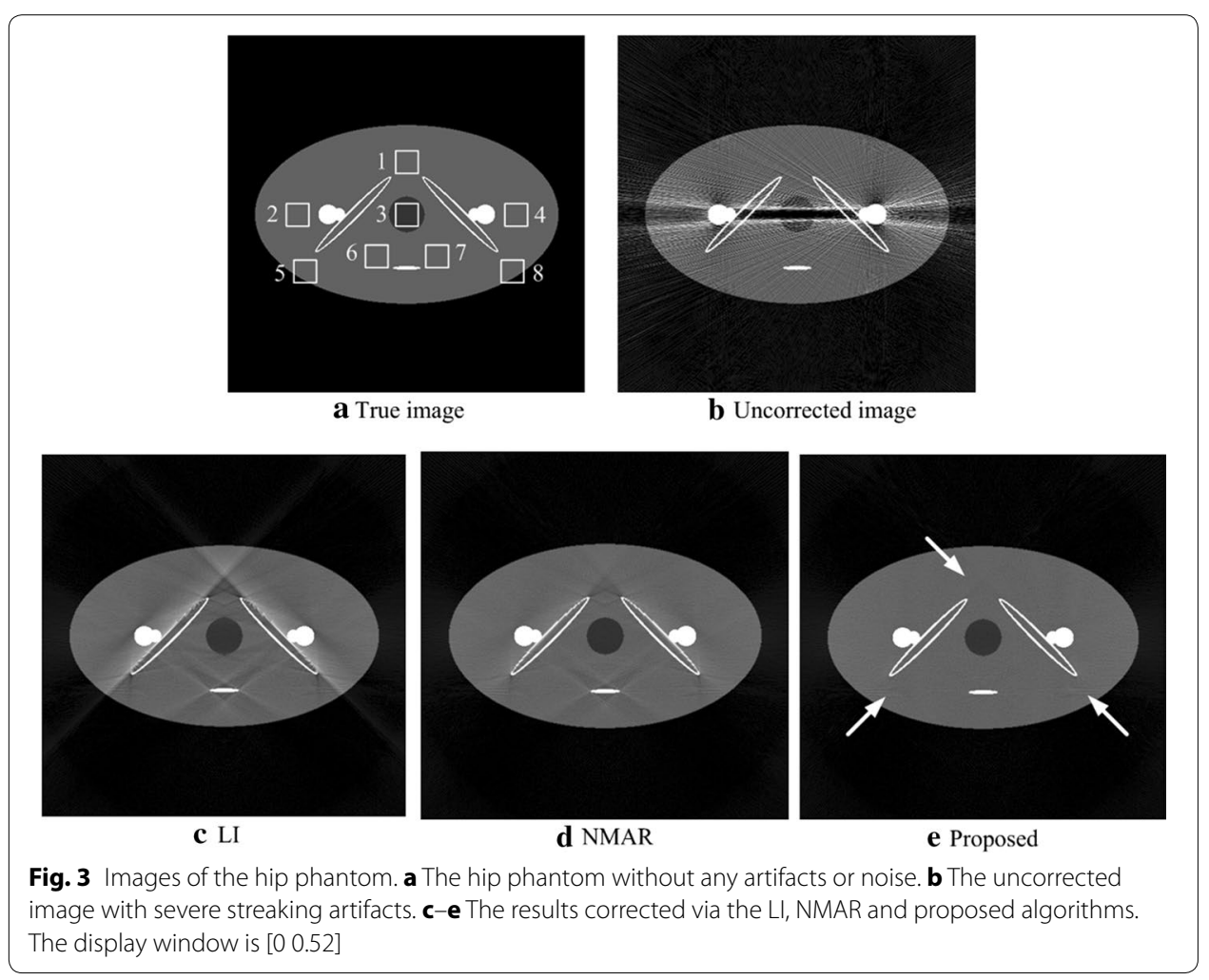


the arrows in Fig. 3e. Thus, from subjective judgment, the proposed algorithm performs the best.

Table 3 gives the quantitative evaluation of the hip phantom results. In this table, the proposed algorithm NMAD is smaller than LI and NMAR, decreasing by 2.93 and $0.51 \%$, respectively, revealing that the image corrected via the proposed algorithm has a better representation for the true image compared with the other two algorithm results. The SNR of the proposed algorithm is larger than LI and NMAR,increasing by 9.35 and $1.04 \mathrm{~dB}$, respectively, implying the proposed algorithm leads to the best corrected image among the three MAR algorithms.

Figure 4 shows the mean pixel value and the standard deviation of eight ROIs in resulted images for the two phantoms. The ROIs of different phantoms are defined in Figs. 2a and 3a. Generally, the proposed method has the mean pixel that is closest to the ideal, and it also has the smallest standard deviation in most ROIs. The results imply that the proposed algorithm has the best performance in suppressing the artifacts.

These two groups of phantom experiments show that whether the bone structure in the image is simple or complex, the proposed algorithm has a good effect on suppressing metal artifacts and reducing the noise level.

Figure 5 illustrates the correction results for the patient with a single metallic dental filling. Figure $5 \mathrm{a}$ is the uncorrected image. Figure $5 \mathrm{~b}-\mathrm{e}$ are the corrected images produced by the LI, NMAR and proposed algorithms. Figure $5 \mathrm{e}-\mathrm{h}$ are the images after local amplification of the corresponding Fig. 5a-d in the ROI; this operator is beneficial for observing the image quality in ROI clearly, and the ROI is defined in Fig. 5a with a white rectangle. In the LI MAR result, the image shows severe secondary artifacts, and some structures are even more blurred. Both NMAR and the proposed algorithm successfully reduce the artifacts and introduce hardly any secondary artifacts, but the proposed algorithm performs better. As indicated by the arrows in Fig. 5g, the residual dark artifacts of the NMAR result are more than those of the proposed one. Thus, in this dataset, the proposed method performs the best, and the performance has proven that the algorithm can be used in complicated bone structure images containing a single implanted metal.

Figure 6 shows the corrections for the patient with two metallic dental fillings. Figure $6 \mathrm{a}-\mathrm{d}$ are the uncorrected images and results corrected via the LI, NMAR and proposed method. Figure $6 \mathrm{e}-\mathrm{h}$ are the local amplifications of the corresponding Fig. $6 \mathrm{a}-\mathrm{d}$ in the ROI, and the ROI is defined in Fig. 6a by the white rectangle. In this dataset, the LI reduces the original metal artifacts existing in the uncorrected image but introduces severe secondary artifacts, making the image quality even lower than that of the uncorrected image. Compared with LI, NMAR produces a better result and does not cause artifacts between teeth; however, secondary artifacts still blur the structures between the two metals. While the result produced via the proposed algorithm reduces the metal artifacts without causing any obvious secondary artifacts, the tissue structures between

Table 3 Quantitative evaluation of the experimental results for the hip phantom

\begin{tabular}{|c|c|c|c|c|c|c|c|}
\hline \multicolumn{2}{|c|}{ Uncorrected } & \multicolumn{2}{|l|}{$\mathrm{LI}$} & \multicolumn{2}{|l|}{ NMAR } & \multicolumn{2}{|l|}{ Proposed } \\
\hline SNR (dB) & NMAD (\%) & SNR (dB) & NMAD (\%) & SNR (dB) & NMAD (\%) & SNR (dB) & NMAD (\%) \\
\hline 12.91 & 56.05 & 13.34 & 18.04 & 21.65 & 15.62 & 22.69 & 15.11 \\
\hline
\end{tabular}




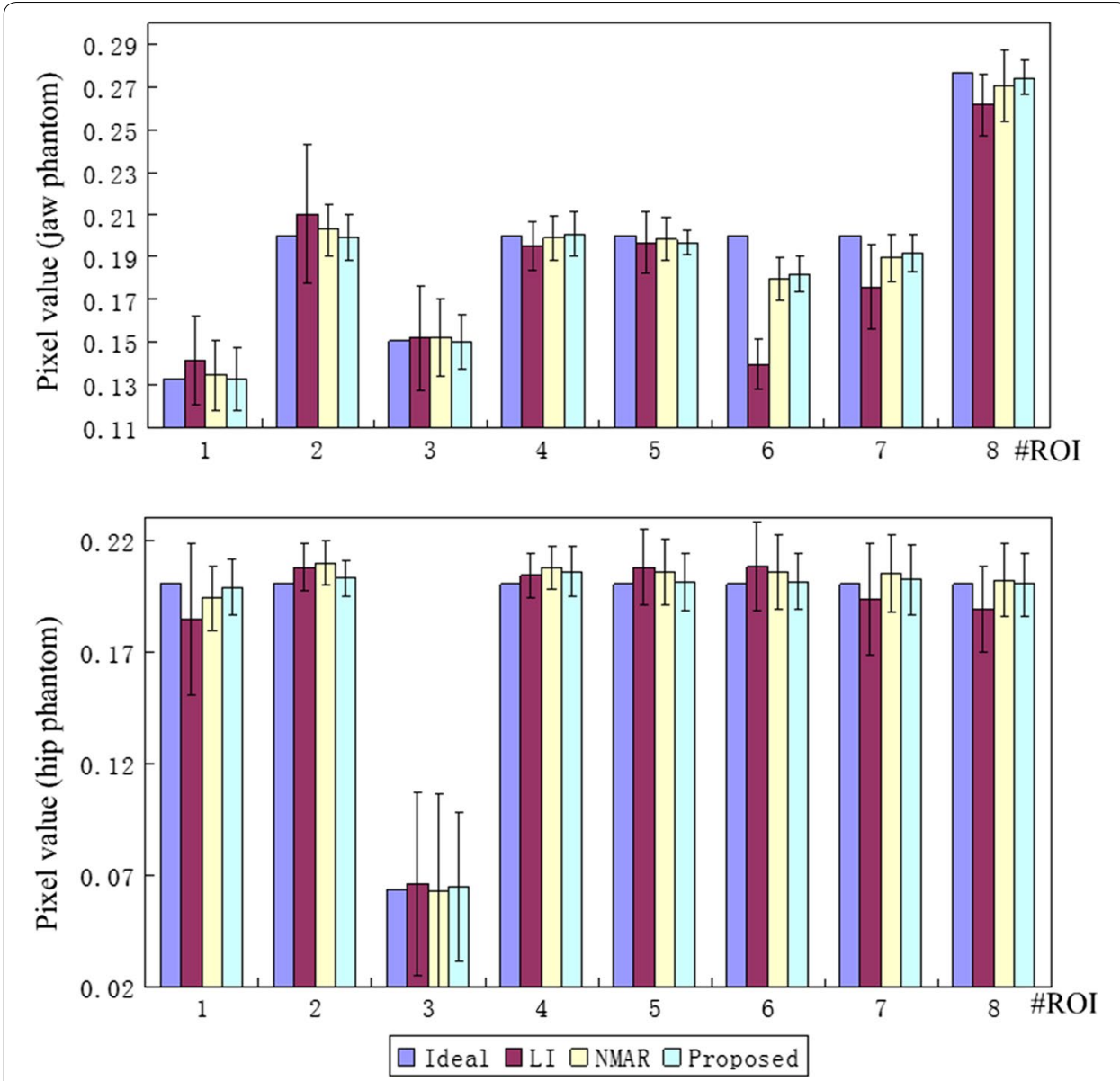

Fig. 4 The mean pixel value and the standard deviation in different ROls for the two phantoms. The top one are the results of the jaw phantom and the bottom one belong to the hip phantom. Eight RIOs are defined in Figs. 2a and 3 a for two phantoms, and each ROI contains $20 \times 20$ pixels

the two metals and around the teeth are very clear. Thus, in this dataset, the proposed method has the best performance, demonstrating that the proposed algorithm has a good effect on reducing the artifacts under the condition of two metal fillings in a complicated bone structure image.

Figure 7 shows the correction results for the patient with a single metallic hip prosthesis. Figure $7 \mathrm{a}$ is the uncorrected image, and Fig. $7 \mathrm{~b}-\mathrm{d}$ are the results corrected via LI, NMAR and the proposed methods. Figure $7 \mathrm{e}-\mathrm{h}$ are local amplifications of the corresponding Fig. 7a-d in the ROI; the ROI is defined in Fig. 7a by the white rectangle. In this dataset, to analyze the quality of the images produced by these MAR methods, we have not implanted metal into the results. For the LI result, as indicated by the arrow in Fig. $7 f$, the region around the metal is totally blurred; in addition, some secondary artifacts are also introduced. Altogether, for the results from the NMAR and proposed methods, they both reduce metal artifacts thoroughly and cause no new artifacts. There are two main reasons for this. First, both the NMAR and proposed methods use the same prior image, implying the proposed method relies on the prior image to some extent. Second, the uncorrected image has a simple structure, which determines that the 


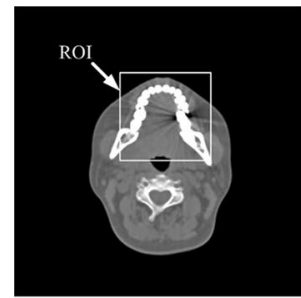

$\mathbf{a}$

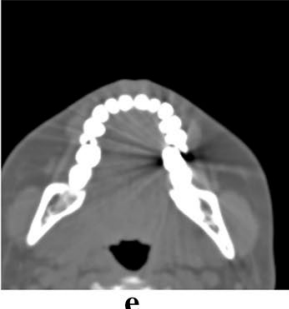

e

Uncorrected image

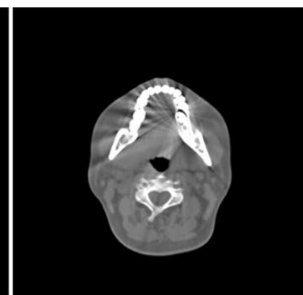

b

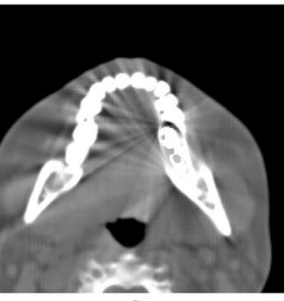

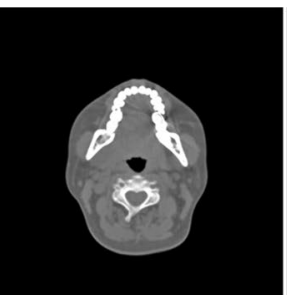

c

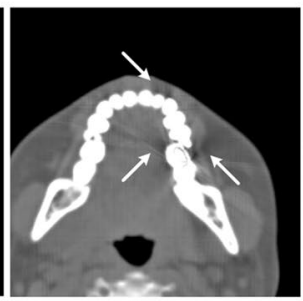

g

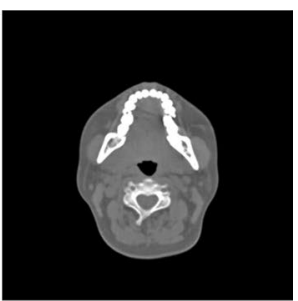

d

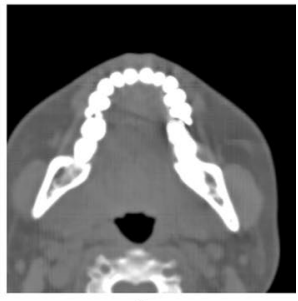

h

Fig. 5 Uncorrected image and results corrected via the different algorithms for the patient with a single metal dental filling. $\mathbf{e}-\mathbf{f}$ The local amplifications of the corresponding images $(\mathbf{a})-(\mathbf{d})$. The display window width and window center are 1200 and $100 \mathrm{HU}$, respectively

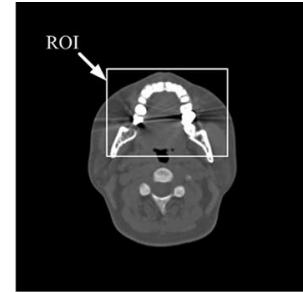

a

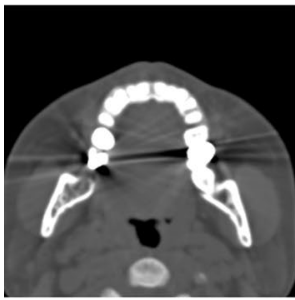

e

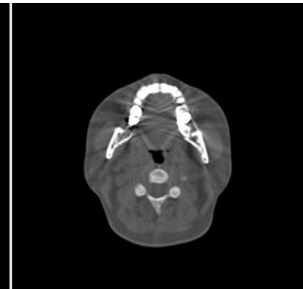

b

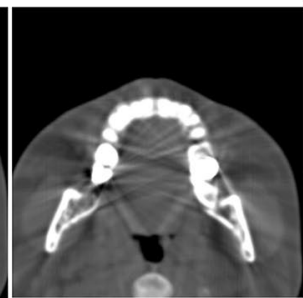

f

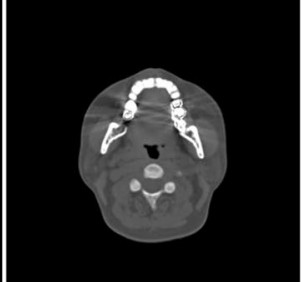

c

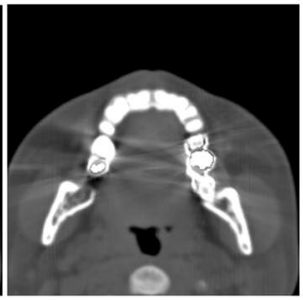

g

NMAR

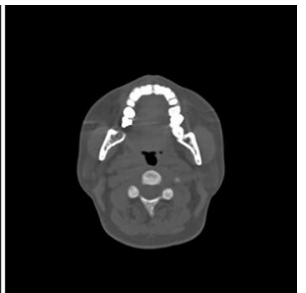

d

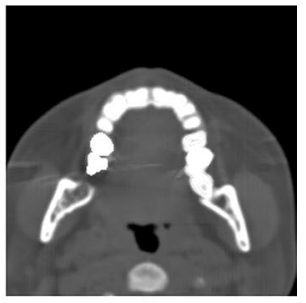

h

Fig. 6 Uncorrected image and results corrected via the different algorithms for the patient with doublemetal dental fillings. e-f The local amplifications of the corresponding images (a)-(d). The display window width and window center are 1600 and $300 \mathrm{HU}$, respectively

metal artifacts is easy to be corrected. On the other hand, due to the uncertainty of the prior image with the method of [14], the prior image of this experiment just makes the NMAR have the same good result as the proposed algorithm. The combination with the previous three groups of experimental results of the NMAR shows that the proposed method is more stable and lower requirement for prior image quality compared to the NMAR. In the next dataset, there are complex bones and soft tissues in the uncorrected image, indicating that the correction of this dataset is the most challenging. 


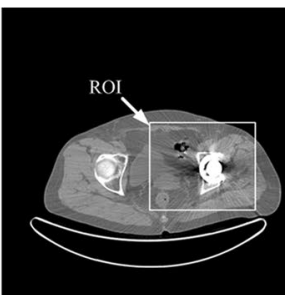

$\mathbf{a}$

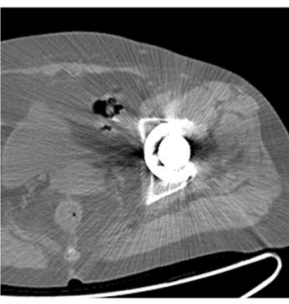

e

Uncorrected image

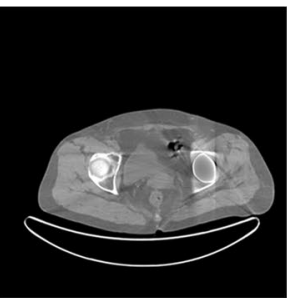

b
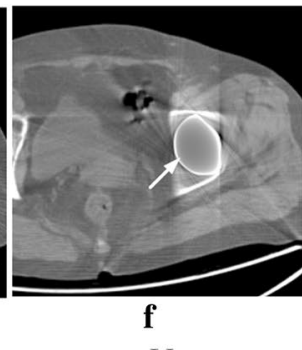

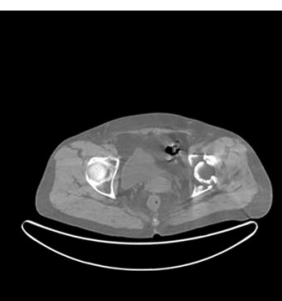

c

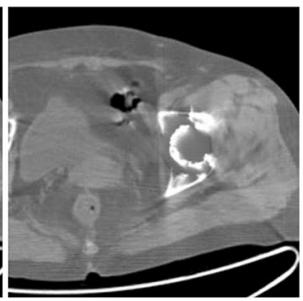

g

NMAR

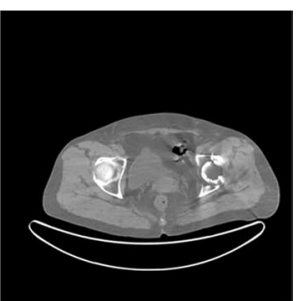

d

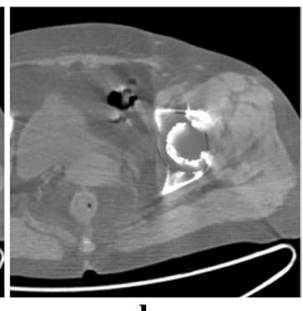

h

Proposed

Fig. 7 Uncorrected image and results corrected via the different algorithms for the patient with a single metallic hip prosthesis. e-f The local amplifications of their corresponding images (a)-(d). The display window width and window center are 800 and $0 \mathrm{HU}$, respectively

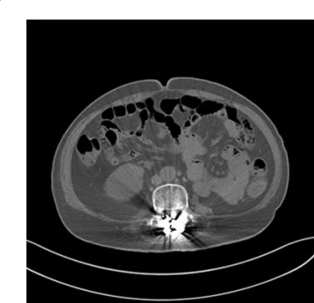

a

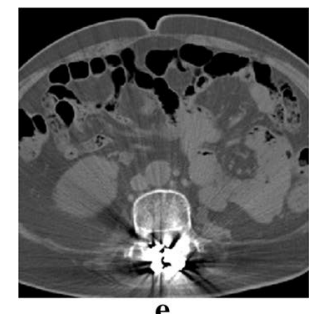

Uncorrected image

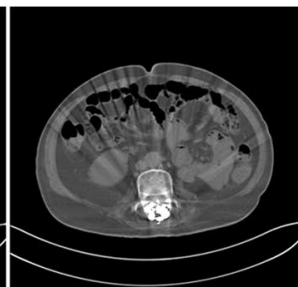

b

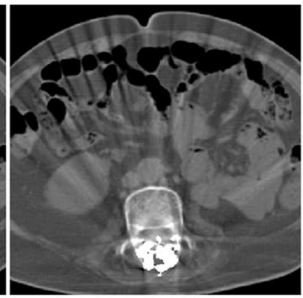

f

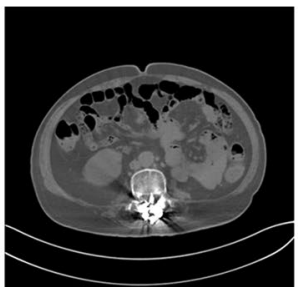

c

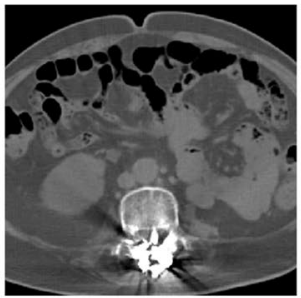

g

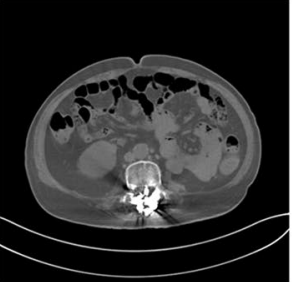

d

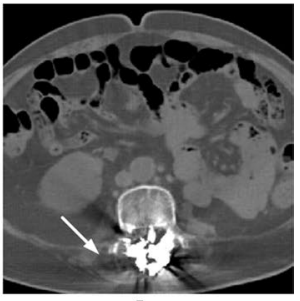

h

Fig. 8 The uncorrected image and the corrected results via the different algorithms for a patient with multiple metallic implants in the vertebrae. e-f The corresponding images after amplifying (a)-(d) 1.6 times. The display window width and window center are 800 and $100 \mathrm{HU}$, respectively

The correction results from the most challenging dataset are shown in Fig. 8. This dataset is from a patient whose vertebrae are implanted with several metals. Figure $8 \mathrm{a}$ is the uncorrected image, which shows severe streaking dark artifacts around the metals and tissue structures that are very complicated, indicating that the process of MAR is not easy. Figure $8 \mathrm{~b}-\mathrm{d}$ are the results corrected via the LI, NMAR and proposed methods. After magnifying Fig. 8a-d 1.6 times, the corresponding images are obtained, Fig. $8 \mathrm{e}-\mathrm{h}$. Here, the LI method reduces the dark metal artifacts obviously, but it brings 
new streak-like dark artifacts and blurs the bone structure around the metals seriously. The NMAR and proposed methods protect the bone structures around the metals effectively and do not introduce new artifacts, but there are still some dark artifacts left. When compared with the NMAR algorithm, the proposed method has some improvements, there are less residual dark artifacts in the position as indicated by the arrow in Fig. 8h. Globally, in this dataset, the proposed algorithm performs better than LI and NMAR in suppressing metal artifacts. As mentioned above, this dataset has complicated structures, indicating that the uncorrected image harbors extremely severe metal artifacts, and the prior image quality will be poor. However, the best performance in this study proves the advantage of the proposed method for complicated bone and tissue structure images.

\section{Discussions}

Metallic implants in the bodies of patients can lead to severe streaking artifacts, therefore degrading the quality of $\mathrm{CT}$ images and impacting the diagnostic procedure by obscuring image details. In this study, we propose a Gaussian diffusion sinogram inpainting algorithm to reduce the metal artifacts in a fan-beam scanner. In our work, the optimization objective is to minimize the prior energy. The Gaussian function is applied to diffuse prior energy, and the prior image is used to define the diffusion position and promote the sparsity of the original image. The gradient descent approach is applied to solve this problem. The performance of the proposed method is compared with two conventional MAR algorithms (LI, NMAR) using both simulation and clinical datasets. In the implementation of the proposed method and NMAR, we use the same prior image produced by the tissue-classified technique. From the results described in the last section, the proposed algorithm has a great advantage overall. However, there are still some factors impacting the effect of the proposed method.

First, the prior image quality is an important influential factor. In this study, the prior image is obtained by the tissue-classified method and often cannot introduce high-quality prior images because of inaccurate classification. Under this background, the proposed algorithm cannot produce an ideal corrected image at all times. This phenomenon reveals that the performance of the proposed method is highly dependent on the prior image. Thus, we may focus our next work on computing a high-quality prior image.

The second factor is the iteration numbers. The number of iterations determines the diffusion degree of the corrupted projections energy. Obviously, under the constraint of the prior image, when there is more energy diffused a better image quality will be obtained. The influence of the iteration times on the image quality is illustrated in Fig. 8.

In Fig. 9, we chose the clinical hip dataset to explain the impact of the iteration numbers because this dataset has an obvious metal region in the projection domain, which is convenient to observe the change in the sinogram. From Fig. 9a-g, with an increase in iteration number we can easily find that the metal projection energy is gradually decreased, the metal artifacts existing in the corresponding images are decreased, and the image quality is improved. However, too many iterations will cause an increase in the correction time. Thus, in this work, to balance the image quality and corrected time, we set the stop criterion as $\| x^{k+1}-x^{k}|| /|| x^{k}||<\eta, \eta=1 \times 10^{-4}$. 


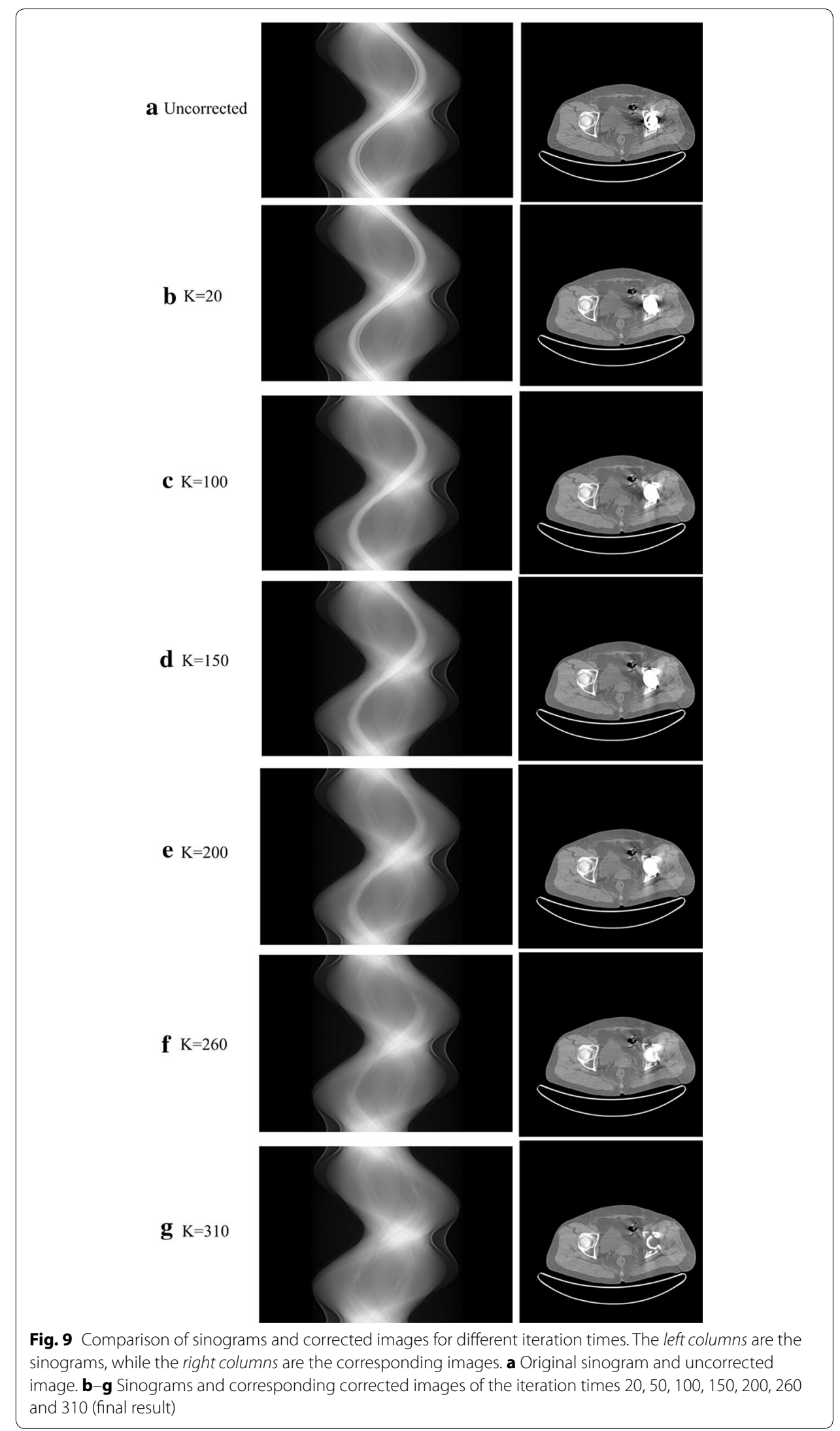




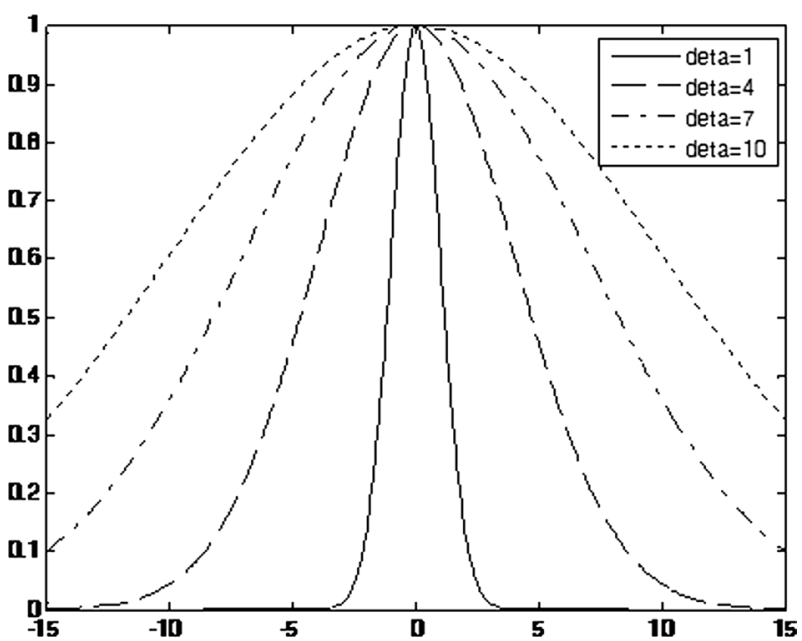

Fig. 10 The Gaussian function curves for different $\delta$ values (deta in the picture)

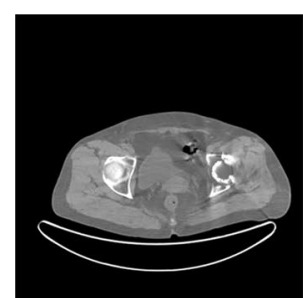

a $\delta=1$

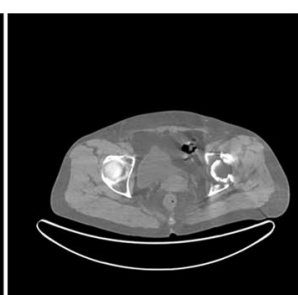

b $\delta=4$

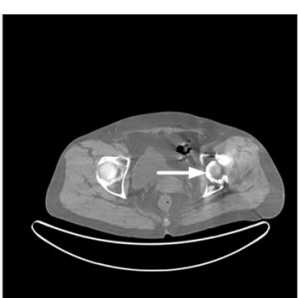

c $\delta=7$

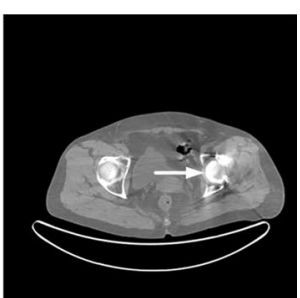

d $\delta=10$

Fig. 11 The results for different $\delta$ values. In $\mathbf{a}-\mathbf{d}$, the value of $\delta$ is set to $1,4,7,10$

The last key factor is the parameter $\delta$ in the Gaussian diffusion function, which determines the final diffusion extent and impacts the corrected image further. Figure 10 shows the Gaussian function with different $\delta$. We can find that, as the value of $\delta$ becomes larger, the diffusion effect of the Gaussian function is better. Figure 11 shows the final corrected images for different $\delta$ values.

Figure 11 shows the corrected results for different $\delta$ values $(1,4,7,10)$. From the picture, Fig. 11a, b almost show the same results because the small $\delta$ values diffuse the energy slowly, and the optimal solution is easy to obtain. However, if the value of $\delta$ is too small, the iteration times would markedly increase. For example, when $\delta=1$, the iterative numbers would be $k=2000$, which is a great waste of time. Conversely, as indicated by the arrows in Fig. 11c, d, the bone structure is not clear compared with (b). As mentioned before, the diffusion process can be observed as a gradient descent, so a large $\delta$ usually cannot diffuse energy to the minimum. Through numerous experiments such as this, we choose $\delta=4$.

\section{Conclusions}

In this study, a Gaussian diffusion sinogram inpainting MAR algorithm is proposed, and the algorithm is realized via gradient descent. In this work, the prior image is obtained by a tissue-classified technique to improve the sparsity of the subtraction sinogram 
and to locate the diffusion position. The metal-only image is obtained by the simple thresholding method. The final corrected image is reconstructed by FBP after sinogram inpainting. The performance of the proposed algorithm is compared with that of the LI and NMAR algorithms using both simulation and clinical studies. Among the simulation and clinical studies, the proposed algorithm reduces the metal artifacts more effectively than the other two representative algorithms. The good performance of the proposed algorithm in several experiments proves that the method can be used in all types of clinical situations stably. However, the proposed algorithm relies on a prior image to some extent; thus, in our next work, we may use deep learning to compute high-quality prior images.

\begin{abstract}
Abbreviations
CT: computed tomography; FBP: filtered back-projection; MAR: metal artifact reduction; LI: linear interpolation; NMAR: normalized metal artifact reduction; LS: least squares; ART: algebraic reconstruction technique; POCS: projection onto the convex set; SNR: signal-to-noise ratio; NMAD: normalized the mean absolute deviation; ROI: region of interest.
\end{abstract}

\title{
Authors' contributions
}

Study concept and design (CP and $\mathrm{ML}$ ); drafting of the manuscript (CP); critical revision of the manuscript for important intellectual content (CP, BQ and JZ); obtained funding (JZ and ML); administrative, technical, and material support (CP, JZ and $\mathrm{BQ})$; polish language and study supervision (CZ, ZW and $\mathrm{YG}$ ). All authors read and approved the final manuscript.

\section{Author details}

${ }^{1}$ Center for Biomedical Engineering, Department of Electronic Science and Technology, University of Science and Technology of China, Hefei, China. ${ }^{2}$ Medical Imaging Department, Suzhou Institute of Biomedical Engineering and Technology, Chinese Academy of Science, Suzhou, China. ${ }^{3}$ PET Center, Huashan Hospital, Fudan University, Shanghai, China.

\section{Acknowledgements}

The authors would like to thank the PET Center, Huashan Hospital, Fudan University for providing the clinical CT images. The authors are also grateful to the anonymous reviewers for their valuable comments and suggestions.

\section{Competing interests}

The authors declare that they have no competing interests.

\section{Availability of data and materials}

All of the datasets related to the current study are available from the corresponding author on reasonable request.

\section{Consent for publication}

In this work, all of the experiments were agreed upon by the patients and the PET Center, Huashan Hospital, Fudan University, Shanghai, China. Additionally, all the datasets can be published publicly.

\section{Ethics approval and consent to participate}

The ethical approval was given by the Medical Ethics Committee of Suzhou Institute of Biomedical Engineering and Technology, Chinese Academy of Science, Suzhou, China. According to the report, the study was permitted to be developed.

\section{Funding}

This work was supported in part by the National Natural Science Foundation of China (No. 61201117), the National Program on Key Research and Development Project (Nos. 2016YFC0104500, 2016YFC0104505, 2016YFC0103500 and 2016YFC0103502), the Natural Science Foundation of Jiangsu Province (No. BK20151232), the Science and Technology Program of Suzhou (No. ZXY2013001), and the Youth Innovation Promotion Association CAS (No. 2014281).

Received: 4 September 2016 Accepted: 4 December 2016

Published online: 05 January 2017

\section{References}

1. Herman GT. Demonstration of beam hardening correction in computed tomography of the head. J Comput Assist Tomogr. 1979;3(3):373-8.

2. Brooks RA, Di CG. Beam hardening in X-ray reconstructive tomography. Phys Med Biol. 1976;21(3):390-8.

3. Pan X, Sidky EY, Vannier M. Why do commercial CT scanners still employ traditional, filtered back-projection for image reconstruction? Inverse Probl. 2009;25(12):123009.

4. Kalender WA, Hebel R, Ebersberger J. Reduction of CT artifacts caused by metallic implants. Radiology. 1987;164(2):576-7.

5. Abdoli M, Ay MR, Ahmadian A, et al. Reduction of dental filling metallic artifacts in CT-based attenuation correction of PET data using weighted virtual sinograms optimized by a genetic algorithm. Med Phys. 2010;37(12):6166-77. 
6. Bazalova M, Beaulieu L, Palefsky S, et al. Correction of CT artifacts and its influence on Monte Carlo dose calculations, Med Phys. 2007;34(6):2119-32.

7. Zhao $S$, Robeltson DD, Wang G, et al. X-ray CT metal artifact reduction using wavelets: an application for imaging total hip prostheses. IEEE Trans Med Imag. 2000;19(12):1238-47.

8. Xue H, Zhang L, Xiao Y, et al. Metal artifact reduction in dual energy $C T$ by sinogram segmentation based on active contour model and TV inpainting. In: Nuclear science symposium conference record (NSS/MIC), 2009 IEEE. IEEE; 2009. pp. 904-908.

9. Duan X, Zhang L, Xiao Y, et al. Metal artifact reduction in CT images by sinogram TV inpainting. In: Nuclear science symposium conference record, 2008. NSS'08. IEEE. IEEE; 2008. pp. 4175-4177.

10. Zhang Y, Pu YF, Hu JR, et al. A new CT metal artifacts reduction algorithm based on fractional-order sinogram inpainting. J X-ray Sci Technol. 2011;19(3):373-84.

11. Chen Y, LiY, Guo H, et al. CT Metal artifact reduction method based on improved image segmentation and sinogram in-painting. Math Probl Eng. 2012;2012(1024-123X):245-55.

12. Tang Z, Hu G, Zhang H. Efficient metal artifact reduction method based on improved total variation regularization. J Med Biol Eng. 2014;34(3):261-8.

13. Bal M, Spies L. Metal artifact reduction in CT using tissue-class modeling and adaptive prefiltering. Med Phys. 2006;33(8):2852-9.

14. Meyer E, Raupach R, Lell M, et al. Normalized metal artifact reduction (NMAR) in computed tomography. Med Phys. 2010;37(10):5482-93.

15. Björck Å. Numerical methods for least squares problems, vol. 31. Philadelphia: Society for Industrial and Applied Mathematics; 1996. p. 408.

16. Beck A, Teboulle M. A fast iterative shrinkage-thresholding algorithm for linear inverse problems. SIAM J Imag Sci. 2009;2(1):183-202.

17. Mehranian A, Ay MR, Rahmim A, et al. 3D prior image constrained projection completion for X-ray CT metal artifact reduction. IEEE Trans Nucl Sci. 2013;60(5):3318-32.

18. Perona P, Malik J. Scale-space and edge detection using anisotropic diffusion. IEEE Trans Pattern Anal Mach Intell. 1990;12(7):629-39.

19. Mohimani H, Babaie-Zadeh M, Jutten C. A fast approach for overcomplete sparse decomposition based on smoothed norm. IEEE Trans Signal Process. 2009;57(1):289-301.

20. Hyder MM, Mahata K. An improved smoothed approximation algorithm for sparse representation. IEEE Trans Signal Process. 2010:58(4):2194-205.

21. Boyd S, Vandenberghe L, Faybusovich L. Convex optimization. IEEE Trans Autom Control. 2006;51 (11):1859.

22. Li M, Zheng J, Zhang T, et al. A prior-based metal artifact reduction algorithm for X-ray CT. J X-ray Sci Technol. 2015;23(2):229-41.

23. La Rivière PJ, Bian J, Vargas PA. Penalized-likelihood sinogram restoration for computed tomography. IEEE Trans Med Imag. 2006;25(8):1022-36.

Submit your next manuscript to BioMed Central and we will help you at every step:

- We accept pre-submission inquiries

- Our selector tool helps you to find the most relevant journal

- We provide round the clock customer support

- Convenient online submission

- Thorough peer review

- Inclusion in PubMed and all major indexing services

- Maximum visibility for your research

Submit your manuscript at www.biomedcentral.com/submit 